\title{
BHLH Domain
}

National Cancer Institute

\section{Source}

National Cancer Institute. bHLH Domain. NCI Thesaurus. Code C13450.

The bHLH domain is a bipartite DNA-binding domain containing a basic region upstream of an amphipathic helix-loop-helix region. The basic region interacts with DNA and the HLH motif mediates protein dimerization. 